University of Nebraska - Lincoln

DigitalCommons@University of Nebraska - Lincoln

Architectural Engineering -- Faculty Publications

Architectural Engineering and Construction,

Durham School of

3-2006

\title{
A Review of Diffuse Reflections in Architectural Acoustics
}

Jonathan Rathsam

University of Nebraska - Lincoln

Lily M. Wang

University of Nebraska - Lincoln, lwang4@unl.edu

Follow this and additional works at: https://digitalcommons.unl.edu/archengfacpub

Part of the Architectural Engineering Commons

Rathsam, Jonathan and Wang, Lily M., "A Review of Diffuse Reflections in Architectural Acoustics" (2006). Architectural Engineering -- Faculty Publications. 14.

https://digitalcommons.unl.edu/archengfacpub/14

This Article is brought to you for free and open access by the Architectural Engineering and Construction, Durham School of at DigitalCommons@University of Nebraska - Lincoln. It has been accepted for inclusion in Architectural Engineering -- Faculty Publications by an authorized administrator of DigitalCommons@University of Nebraska Lincoln. 


\title{
A Review of Diffuse Reflections in Architectural Acoustics
}

\author{
Jonathan Rathsam and Lily M. Wang
}

Architectural Engineering Program, University of Nebraska -Lincoln, Peter Kiewit Institute, 1110 S. $67^{\text {th }}$ St., Omaha, NE 68182-0681, PH (402) 554-2038; FAX (402) 554-2080; email: jrathsam@mail.unomaha.edu

\section{Abstract}

Diffuse reflections have received increasing attention in architectural acoustics over the past quarter century. The term diffuse refers to non-specular directions. This paper identifies three mechanisms that cause diffuse reflections. Practitioners have found that changing specular reflections into diffuse reflections can reduce noise and echoes both indoors and outdoors. Developers of acoustical modeling software have also found that modeling diffuse in additional to specular reflections produces more accurate predictions. The final portion of this paper presents results for a study of scattering coefficients in acoustical modeling software.

\section{Introduction: The Mechanisms of Diffuse Reflections}

Sound energy that reflects from a boundary is classified as either specular or diffuse. A specular reflection, like light reflecting from a mirror, bounces off the surface with the same angle as it encountered the surface. A diffuse reflection occurs when the sound energy is scattered into non-specular directions. Three mechanisms of diffuse reflection are introduced below.

A clear light bulb transmits light directly from the filament through the glass to objects beyond the bulb. In a frosted light bulb, however, many tiny irregularities in the glass cause the light to be spread into all directions upon exiting the bulb. In the same way, the roughness of an acoustical reflecting surface causes acoustical waves to be spread out in all directions. Surface roughness is the first mechanism of diffuse reflection.

The second mechanism of diffuse reflection is edge diffraction. Edge diffraction accounts for a person's ability to hear a neighbor speaking on the other side of a brick wall even though the neighbor cannot be seen. Edge diffraction can be visualized as follows: at the boundary between the brick wall and the air at the top of the wall, new secondary sources are formed that radiate sound spherically into the space around them (see Figure 1). In this way, a straight-line path from the listener's 
ear to a sound source at the top of the wall is created. In room acoustics, edge diffraction occurs whenever a sound wave encounters a change in the reflecting surface. This can be a change in material, such as brick to air, or a change the orientation of surfaces, such as at the boundary between a wall and the ceiling.

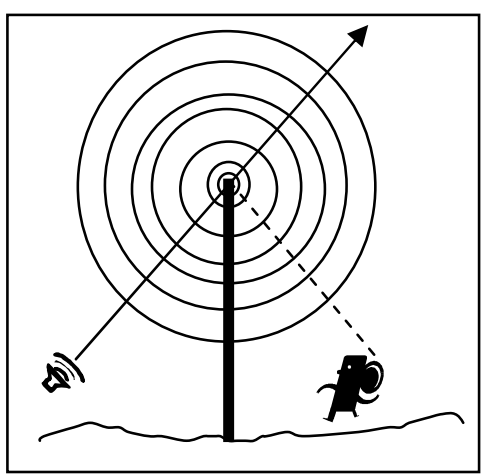

Figure 1. A sound wave undergoing edge diffraction creates a secondary source at the boundary between the brick wall and the air.

A third mechanism of diffuse reflections is a wall treatment called a numerical diffuser. The mechanism of diffuse reflections for numerical diffusers is neither surface roughness, nor edge diffraction. A numerical diffuser is comprised of wells of equal width with varying depth. Incident sound travels down the wells and reemerges from each well with a different phase. The well openings then become individual sound sources, which combine with one another to produce reflections in nonspecular directions (see Figure 2). This is the third mechanism of diffuse reflections.

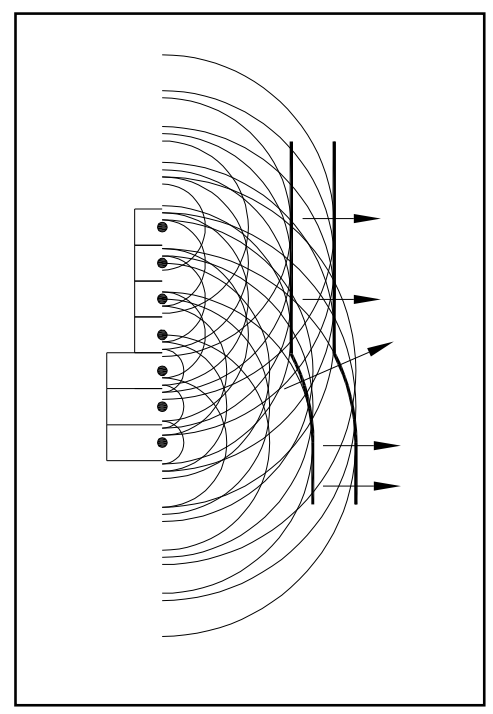

Figure 2. In a numerical diffuser, wells of different depths produce non-specular reflections. In this figure, the incident sound was a plane wave, normally incident on this (simplified) numerical diffuser (after D'Antonio and Cox, 2003). 


\section{Practical Applications of Diffusers}

Increasing the diffusion of room surfaces such as walls or ceilings by increasing surface roughness or adding numerical diffusers can help to avoid unwanted acoustical phenomena. This section provides examples of such situations.

In auditoria, two surface treatments can be applied to control annoying echoes. The first is an absorber, and the second is a diffuser. An absorber reduces the overall reflection, removing sound energy from the auditorium. A diffuser will spread that reflection into all directions, reducing the strength of the specular reflection and echo, but preserving the sound energy within the space. Controlling the echo while preserving the energy is preferable for unamplified performances because sound energy in the space is limited.

Another unwanted acoustical phenomenon in auditoria is comb filtering. Comb filtering occurs when a signal interferes with itself. For example an early specular reflection from a rigid sidewall can interfere with the direct sound at a listener's ears. The resulting spectrum has many dips occurring at frequencies where arriving wavefronts cancel each other (see Figure 3). A diffuser will lengthen the time interval over which a reflection arrives at a listener. This change in time envelope also changes the reflection's spectral content, making it dissimilar from the direct sound. The result of decreasing similarity between the direct sound and reflection is less comb filtering.

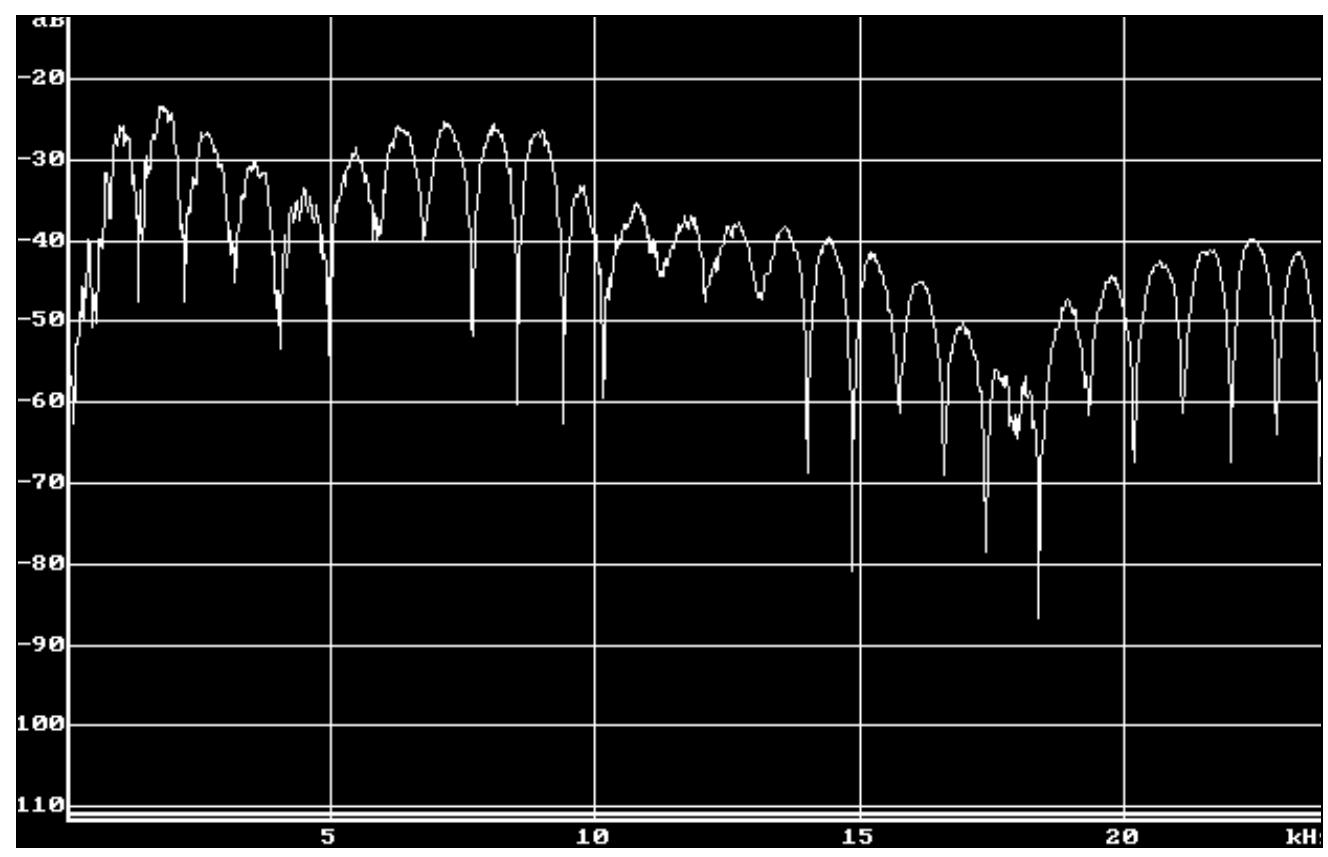

Figure 3. This periodic spacing of dips on a logarithmic frequency (horizontal) axis is a standard comb filter. It is called a comb filter because the regularly spaced dips resemble the teeth of a comb. 
There are also outdoor applications for diffusers. Road side barriers, for example, are a common solution to the problem of traffic noise. While these barriers generally block the direct path between traffic and listeners, there can be a reflection from the barrier over the vehicle to listeners on the other side of the road (Figure 4b). Another problem is a double reflection from the barrier to the side of a truck and then over the wall (Figure 4a). Absorbers are not recommended for this outdoor application because they are susceptible to weather damage. A diffuser, however, can be fashioned out of weather resistant materials and installed to spread out the reflection, reducing the level of the specular reflection.

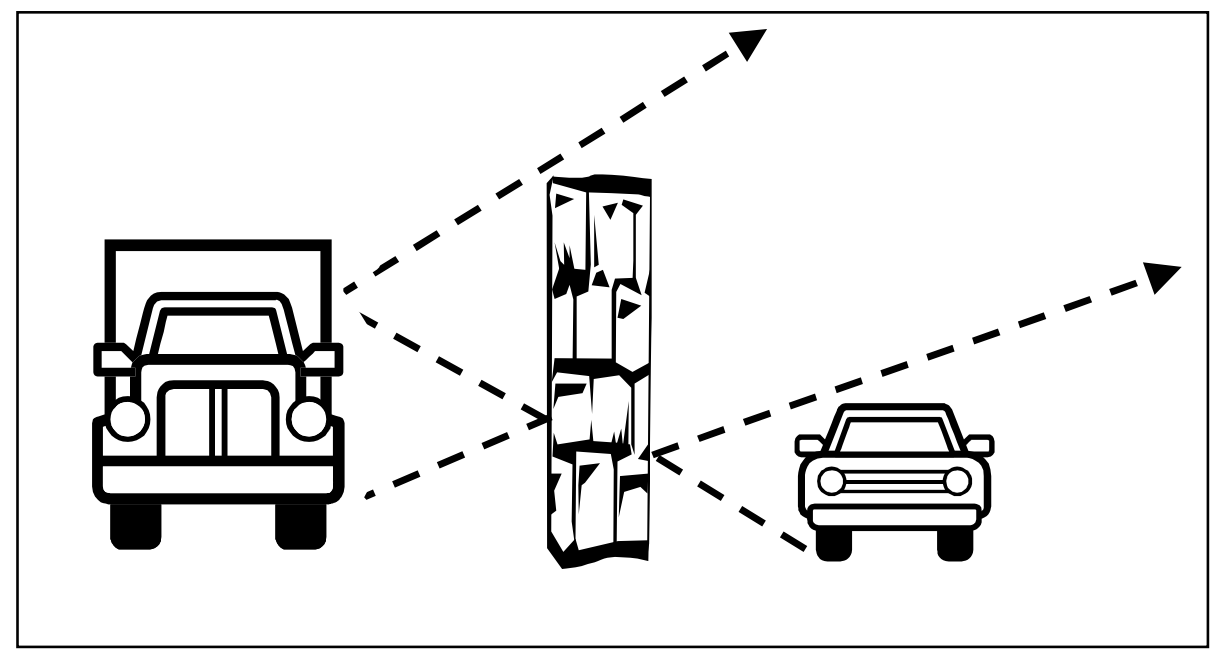

Figures 4a (left) and 4b (right). These illustrations show how strong specular reflections from roadside barriers for both cars and trucks can still cause noise problems (after Cox and D'Antonio, 2004).

Another outdoor application for diffusers is a street canyon. Street canyons are streets lined with high building on either side. Traffic noise from the street can be reflected back to the street by balcony fronts, increasing the noise even more.

Weather-resistant diffusers can be used on these balcony fronts to reduce the noise reflected back down to the street.

\section{A Practical Application of Diffuse Reflections: Modeling Surface Roughness}

In the past fifteen years, several commercial programs have become available for modeling room acoustics. The most common algorithms trace rays from a source as they propagate around a user-defined geometry and record the strength and arrival time of rays that pass through a specified receiver location. The energy of each ray is reduced upon reflection from an absorptive surface. The model stops when it has exceeded a user-set response length (time) or number of reflections per ray.

Research has shown that including diffuse reflections leads to increased accuracy in computer modeling. A 1990 study predicting the sound field in large rooms found excellent agreement between prediction and measurement if ceiling and 
walls were made to be $60 \%-80 \%$ diffusely reflecting (Hodgson, 1990). By the year 2000 , nearly all of the world's room acoustic prediction modules accounted for diffuse reflections, or scattering (Bork, 2000).

To model the scattering of real-life materials, there must be a quantifier of scattering. Last year a standard, ISO 17497-1:2004, was released for measuring the scattering from a surface. The measurement procedure described in this standard produces a frequency-dependent value between 0 and 1 that quantifies the proportion of energy reflected by a rough surface into non-specular directions. This value is called a scattering coefficient. This measurement standard holds great promise for improving room acoustic prediction software, but the vast majority of materials and finishes remain to be measured.

In the absence of measured scattering data, it is helpful to develop an intuition for how the amount of scattering influences the various room acoustics parameters. Specifically, which parameters are most sensitive to changes in scattering coefficients? What materiality factors within the room affect a room's sensitivity to changes in scattering coefficients?

To answer these questions, the authors conducted a study in which the scattering coefficients of all surfaces within a model were changed simultaneously from 0 to $0.1,0.3,0.5$, and 0.8 . The values of four room acoustics parameters were recorded at each trial. Other factors were changed, such as the location of acoustical absorption within the room, the disparity between most absorptive and least absorptive surface, and the average absorption value within the room.

The results of this study were that reverberation time (T30) is the parameter most affected by changes in the scattering coefficient. The reverberation time is the time required for a sound in the room to decay to one-millionth of its initial energy. Reverberation time is sensitive to scattering because in a room with parallel reflecting surfaces, sound can get stuck between parallel reflective walls, increasing the reverberation time. Introducing scattering redirects this sound to other more absorptive surfaces in the room. The following flow chart (see Figure 5) ranks room materiality factors in terms of how much they affect the model's sensitivity to scattering coefficients. If the model has no large reflective surfaces that share a surface normal, the only significant factor examined that determines the model's sensitive to changes in scattering coefficient is the average absorption coefficient within the room. If a room does have large reflective surfaces that share a surface normal, then the factors that affect the room's sensitivity, from most to least influential are: area of these reflective surfaces, disparity of absorption, and average absorption coefficient. 


\begin{tabular}{|l|l|}
\hline $\begin{array}{l}\text { Does room have large } \\
\text { reflective surfaces with } \\
\text { common surface normal? }\end{array}$ \\
\hline $\begin{array}{l}\text { Most significant factor } \\
\text { coefficient }\end{array}$ & $\begin{array}{l}\text {-Most significant factor is area of } \\
\text { these reflective surfaces } \\
\text {-Second most significant factor is } \\
\text { disparity of materials } \\
\text {-Third most significant factor is } \\
\text { average absorption }\end{array}$ \\
\hline
\end{tabular}

Figure 5: This flow chart is used to predict model sensitivity to changes in scattering coefficients.

\section{Conclusion}

This paper has introduced some basics of diffuse reflections in architectural acoustics. Three mechanisms of diffuse reflection have been covered: surface roughness, edge diffraction, and numerical diffusers. Several practical uses of diffusers are identified, including use for echo control, reduction of comb filtering, and outdoor application for controlling traffic noise. The paper also discusses how diffuse reflections are quantified for use in room acoustics modeling software.

Results are shared from a study examining the materiality factors that make a room sensitive to changes in scattering coefficient.

Diffuse reflections will play a central role as modeling software is refined in the coming years. One relevant question in acoustical computer modeling is how best to implement edge diffraction in ray-tracing algorithms. Software designers suspect that present-day innacuracies are due to insufficient modeling of edge diffraction. Accurate modeling of edge diffraction is critical, for example, in the case of opera halls where the orchestral sound must diffract around the orchestra pit boundaries to reach the stage and audience. 


\section{References}

Bork, I. (2000). "2nd Round Robin on Room Acoustical Computer Simulations." Acta Acustica 86, 943-956.

Cox, T.J., and D'Antonio, P. (2004). Acoustic absorbers and diffusers: theory, design, and application, Spon, New York, NY, Chap. 2, p. 55.

D'Antonio, P., and Cox, T. (2003) "Technical Bulletin on the Application of Diffusion in Critical Listening Rooms." RPG Diffusor Systems, Inc. Upper Marlboro, MD. Figure 2.

Hodgson, M. (1990). "Evidence of diffuse surface reflections in rooms." J. Acoust. Soc. Am., 89 (2), 765-771.

ISO 17497-1:2004: Acoustics -- Sound-scattering properties of surfaces -- Part 1:

Measurement of the random-incidence scattering coefficient in a reverberation room. 